\title{
ADAPTIVE ENTROPY-CONSTRAINED MATCHING PURSUIT QUANTIZATION
}

\author{
Pierre Vandergheynst and Pascal Frossard
}

\author{
Signal Processing Laboratory \\ Swiss Federal Institute of Technology \\ $\mathrm{CH}-1015$ Lausanne, Switzerland \\ pierre.vandergheynst@epf1.ch
}

\begin{abstract}
This paper proposes an adaptive entropy-constrained Matching Pursuit coefficient quantization scheme. The quantization scheme takes benefit of the inherent properties of Matching Pursuit streams where coefficients energy decreases along with the iteration number. The decay rate can moreover be upper-bounded with an exponential curve driven by the redundancy of the dictionary. An optimal entropy-constrained quantization scheme can thus be derived once the dictionary is known. We propose here to approximate this optimal quantization scheme by adaptive quantization of successive coefficients whose actual values are used to update the quantization scheme parameters. This new quantization scheme is shown to outperform classical exponential quantization in the case of both random dictionaries and practical image coding with Gabor dictionaries.
\end{abstract}

\section{INTRODUCTION}

Non-orthogonal transforms presents several interesting properties which position them as an interesting alternative to orthogonal transforms like DCT or wavelet based schemes. Decomposing a signal over a redundant dictionary improves the compression efficiency, especially at low bit rates where most of the signal energy is captured by only few elements. In this context, Matching Pursuit algorithms [1] provide an interesting way to iteratively decompose the signal in its most important features with a limited complexity. It outputs a stream composed of atoms or basis functions along with their respective coefficients. Since the Matching Pursuit coefficients generally take on real values, quantization is however necessary to reduce the bandwidth needed to transmit them. Quantization errors have been studied in $[2,3]$ in the context of overcomplete frame expansions and consistent Matching Pursuit. This paper focuses particularly on a posteriori coefficient quantization for a general Matching Pursuit decomposition, in contrary to usual schemes $[4,5,6]$ where the encoder uses the quantized coefficients to update the residual signal.

An optimal entropy-constrained quantization scheme can be derived taking benefit of the inherent properties of Matching Pursuit streams [7]. The coefficients energy are indeed upper-bounded by a exponential decay curve along with the iteration number. This curve only depends on the properties of the dictionary and the search algorithm. Hence, the contribution of the Matching Pursuit coefficients to the quantization error clearly depends on their position within the encoded stream, and hence on the redundancy of the dictionary. Based on the characterization of the energy decay curve, the quantization range and the number of quantization steps can be adapted to the relative importance of the coefficients. Moreover, the optimal number of atoms for a given bit budget is also given by the exponential decay curve parameters that depend on the redundancy factor and the signal energy. In this paper, an adaptive but suboptimal quantization scheme is proposed to approximate the optimal entropy-constrained quantization in the practical case of large dictionaries. The actual values of the coefficients are used to update the quantization scheme parameters, thus avoiding estimation error effects due to suboptimal Matching Pursuit search on large size dictionaries. This new adaptive quantization scheme truly outperforms entropy-constrained exponential quantization schemes, especially at low bit rates. Furthermore, it is shown to achieve very good results in the practical case of image compression.

The paper is organized as follows: Section 2 first overviews the Matching Pursuit algorithm and presents the convergence properties of the decomposition. Section 3 then presents an optimal entropy-constrained a posteriori quantization of Matching Pursuit coefficients. A suboptimal adaptive quantization scheme is derived in Sec. 4. Experimental results are given in Sec. 5 for random and Gabor dictionaries in the practical case of image coding. Finally, concluding remarks are given in Section 6 .

\section{MATCHING PURSUIT OVERVIEW}

In contrast to orthogonal transforms, overcomplete expansions of signals are not unique. The number of feasible decompositions is infinite, and finding the best solution under a given criteria is a NP-complete problem. In compression, one is interested in representing the signal with the smallest number of elements, that is in finding the solution with most of the energy on only a few functions. Matching Pursuit [1] is one of the sub-optimal approaches that greedily approximates the solution to this NP-complete problem.

Matching Pursuit (MP) is an adaptive algorithm that iteratively decomposes any function $f$ in the Hilbert space $\mathcal{H}$ in a possibly redundant dictionary of functions called atoms [1]. Let $\mathcal{D}=\left\{g_{\gamma}\right\}_{\gamma \in \Gamma}$ be such a dictionary with $\left\|g_{\gamma}\right\|=1$ and $\Gamma$ represents the set of possible indexes. The function $f$ is first decomposed as follows :

$$
f=\left\langle g_{\gamma_{0}} \mid f\right\rangle g_{\gamma_{0}}+\mathcal{R} f,
$$

where $\left\langle g_{\gamma_{0}} \mid f\right\rangle g_{\gamma_{0}}$ represents the projection of $f$ onto $g_{\gamma_{0}}$ and $\mathcal{R} f$ is a residual component. Since all elements in $\mathcal{D}$ have by definition a unit norm, it is easy to see from Eq. (1) that $g_{\gamma_{0}}$ is orthogonal to 
$\mathcal{R} f$, and this teads to

$$
\|f\|^{2}=\left|\left\langle:_{\gamma_{0}} \mid f\right\rangle\right|^{2}+\|\mathcal{R} f\|^{2} .
$$

To minimize $\|\mathcal{R} f\|$, one must choose $g_{\gamma_{0}}$ such that the projection coefficient $\left|\left\langle g_{\gamma_{0}} \mid f\right\rangle\right|$ is maximum. The pursuit is carried out by applying iteratively the same strategy to the residual component. After $N$ iterations, one has the following decomposition for $f$ :

$$
f=\sum_{n=0}^{N-1}\left\langle g_{\gamma_{n}} \mid \mathcal{R}^{n} f\right\rangle g_{\gamma_{n}}+\mathcal{R}^{N} f
$$

where $\mathcal{R}^{N}$ is the residual of the $N^{t h}$ step with $\mathcal{R}^{0} f=f$. Similarly, the energy $\|f\|^{2}$ is decomposed into :

$$
\|f\|^{2}=\sum_{n=0}^{N-1}\left|\left\langle g_{\gamma_{n}} \mid \mathcal{R}^{n} f\right\rangle\right|^{2}+\left\|\mathcal{R}^{N} f\right\|^{2} .
$$

Although Matching Pursuit places very few restrictions on the dictionary, the latter is strongly related to convergence speed and thus to coding efficiency. Any collection of arbitrarily sized and shaped functions can be used as dictionary, as long as completeness is respected. The completeness property ensures that Matching Pursuit is able to perfectly recover the input signal after a possibly infinite number of iterations.

The convergence speed of Matching Pursuit corresponds to its ability to extract the maximum signal energy in a few iterations. In other words, it corresponds to the decay rate of the residue and thus the coding efficiency of the Matching Pursuit. The approximation error decay rate in Matching Pursuit have been shown to be bounded by an exponential [1,8]. From [9], there exists $\alpha>0$ and $\beta>0$ such that for all $m \geq 0$ :

$$
\left\|\mathcal{R}^{m+1} f\right\| \leq\left(1-\alpha^{2} \beta^{2}\right)^{\frac{1}{2}}\left\|\mathcal{R}^{m} f\right\|,
$$

where $\alpha \in(0,1]$ is an optimality factor. This factor depends on the algorithm that, at each iteration, searches for the best atom in the dictionary. The optimality factor $\alpha$ is set to one when the MP browses the complete dictionary at each iteration. The parameter $\beta$ depends on the dictionary construction. It represents to ability of the dictionary functions to capture features of any input function $f$ and satisfies :

$$
\sup _{\gamma}\left|\left\langle f, g_{\gamma_{n}}\right\rangle\right| \geq \beta\|f\|
$$

The redundancy factor $\beta$ corresponds thus to the cosine of the maximum possible angle between a direction $f$ and its closest direction among all dictionary vectors. A general formulation of the redundancy can be found in [10].

\section{ENTROPY-CONSTRAINED COEFFICIENT QUANTIZATION}

The aim of the quantization is clearly to offer the best possible reconstruction quality for a given bit budget. The quantization error can first be upper-bounded by the sum of the coefficient quantization errors. Indeed, the squared quantization error $D_{Q}$ between the signal approximation $f_{i i}$ and its reconstructed version $\tilde{f}$ can generally be written as :

$$
D_{Q}=\left\|f_{N}-\tilde{f}\right\|^{2}=\left\|\sum_{\lambda_{n}=0}^{N-1} c_{\gamma_{n}} g_{\gamma_{n}}-\sum_{n=0}^{N-1} \tilde{c}_{\gamma_{n}} g_{\widetilde{\gamma_{n}}}\right\|^{2}
$$

where $c_{\gamma_{n}}$ denotes a MP coefficient, or equivalently the inner product $\left\langle g_{\gamma_{n}} \mid \mathcal{R}^{n} f\right\rangle$ and $\tilde{c}_{\gamma_{n}}$ represents the distorted coefficient. Let $\xi_{n}=c_{\gamma_{n}}-\tilde{c}_{\gamma_{n}}$ denote the error on the coefficient. It will be assumed in the remainder of this paper that atom indexes are correctly received, i.e., that $g_{\widetilde{\gamma_{n}}}=g_{\gamma_{n}}$. By triangular inequality, $D_{Q}$ can thus be bounded as

$$
\begin{aligned}
D_{Q} & =\left\|\sum_{n=0}^{N-1}\left(c_{\gamma_{n}}-c_{\gamma_{n}}\right) g_{\gamma_{n}}\right\|^{2} \\
& \leq \sum_{n=0}^{N-1}\left|\xi_{n}\right|^{2}\left\|g_{\gamma_{n}}\right\|^{2} \leq \sum_{n=0}^{N-1}\left|\xi_{n}\right|^{2},
\end{aligned}
$$

since $\left\|g_{\gamma}\right\|=1$. Eq. (8) provides an upper-bound to the reconstruction error due to quantization noise. Finally, the total distortion has also to take into account the signal approximation error due to the finite number of Matching Pursuit iterations. Using Eq. (8) the total distortion can be written as :

$$
\begin{aligned}
D & \leq \sum_{n=0}^{N-1}\left|\xi_{n}\right|^{2}+\left\|\mathcal{R}^{N} f\right\|^{2} \\
& \leq \sum_{n=0}^{N-1}\left|\xi_{n}\right|^{2}+\left(1-\alpha^{2} \beta^{2}\right)^{N}\|f\|^{2},
\end{aligned}
$$

where the energy of the residue at iteration $N$ is bounded thanks to Eq. (5).

The optimal quantization then minimizes the reconstruction error given by Eq. (9) for a given bit budget. Since the quantization error depends on the absolute value of the error on the coefficients, the highest iteration elements can be more coarsely quantized than the first elements for the same contribution to the quantization error. Hence, the exponential upper-bound on the coefficients can be used to design an efficient quantization scheme. Clearly, there is no need to quantize all coefficients on the same range, since their values exponentially decrease. Bits can thus be saved by limiting the quantization region between 0 and the exponential decay curve given by the parameters (i.e., $\beta$ and $\|f\|$ ). An additional bit of sign suffices to completely characterize the coefficients. The number of coefficients, as well as the number of bits per coefficient have now to be optimized in this context of exponentially decaying quantization range.

Assume now in a first approximation that the distribution of the coefficients norm is uniform between 0 and the exponential upper-bound. For complexity reasons, and under the previous assumptions, the coefficient $c_{j}$ is uniformly quantized within the exponentially decaying quantization range

$$
I_{j}=\nu^{j}\|f\|
$$

where $\nu=\left(1-\alpha^{2} \beta^{2}\right)^{\frac{1}{2}}$. Let $n_{j}$ be the number of quantization steps within $I_{j}$ for the quantization of the $j^{\text {th }}$ coefficient. With the previous assumptions, the distortion due to quantization can be written as :

$$
D_{Q} \leq \sum_{n=0}^{N-1}\left|\xi_{n}\right|^{2} \leq \sum_{j=0}^{N-1} \frac{\nu^{2 j}\|f\|^{2}}{12 n_{j}^{2}} .
$$

Note that uniform quantization within an exponentially decaying range is similar to an exponential quantization within the complete range of the coefficients values $[11,12]$. The optimal quantization 
now minimizes the total distortion $D$ for a bit rate $R$, or equivalently, minimizes the bit rate for a given distortion. In other words, we have to find the optimal parameters $n_{j}>1$ and $N$ that minimizes the distortion for a given rate. The Lagrangian multiplier method is well suited for this kind of constrained optimization problems. It defines a cost function $\mathcal{L}(\lambda)$ as the sum of the objective distortion function and the constraint on the rate, weighted by the Lagrangian multiplier $\lambda$. In our case the cost function may be written as :

$$
\begin{aligned}
\mathcal{L}(\lambda) & =D+\lambda R \\
& =\sum_{j=0}^{N-1} \frac{\nu^{2 j}\|f\|^{2}}{12 n_{j}^{2}}+\nu^{2 N}\|f\|^{2}+\sum_{j=0}^{N-1} \lambda\left(\log _{2}\left(n_{j}\right)+B\right),
\end{aligned}
$$

where $B$ represents the average number of bits needed to code the atom index after possible entropy coding. Under the assumption of uniform coefficient distribution, the Lagrangian formulation is equivalent to an entropy-constrained quantization problem. The rate $R$ indeed represents in this case the entropy of the quantized coefficients. The optimal quantization is given by

$$
n_{j}=\sqrt{\frac{\|f\|^{2} \nu^{2 j} \log 2}{6 \lambda}}
$$

and imposes an exponential law to the number of quantization levels :

$$
\frac{n_{j+1}}{n_{j}}=\frac{\nu^{j+1}}{\nu^{j}}=\left(1-\alpha^{2} \beta^{2}\right)^{\frac{1}{2}} .
$$

The complete derivation as well as the optimal number of MP coefficients $N$ are detailed in [7].

\section{ADAPTIVE QUANTIZATION}

The previous optimal quantization scheme has several limitations in practical cases of large dictionaries. Moreover, the accuracy of the exponential upper-bound is highly dependent on the Matching Pursuit search algorithm. To overcome these limitations we now propose a suboptimal though very practical algorithm based on the development of the previous section. The key idea lies in a dynamic computation of the redundancy factor $\beta$ (i.e., the parameter $\nu$ ) from the quantized data, which is the only information available at the decoder. As in the previous section, we only focus on the magnitude of coefficients, reporting their sign on an additional bit.

The adaptive quantization schemes performs as follows. Let $Q\left[c_{k}\right], k=1, \ldots j-1$ denote the quantized counterparts of the $j-$ 1 first coefficients. Due to the exponential decay of the magnitude, coefficient $c_{j}$ is very likely to be smaller than $Q\left[c_{j-1}\right]$. It can thus be quantized in the range $\left[0, Q\left[c_{j-1}\right]\right]$. The number of quantization levels at step $j$ is theoretically driven by the redundancy factor as given by Eq. (14). The adaptive quantization uses an estimate of the redundancy factor to compute the number of quantization levels as :

$$
n_{j}=\bar{\nu}_{j-1} n_{j-1}
$$

The estimate of the redundancy factor $\tilde{\nu}$ is then updated replacing the quantization range $I_{j}$ in Eq. (10) with the previously quantized coefficient $Q\left[c_{j-1}\right]$ as :

$$
\tilde{\nu_{j}}=\left(\frac{Q\left[c_{j}\right]}{\|f\|}\right)^{1 / j}
$$

This can be summarized by Algorithm 1. In practice, it will run as long as there are bits available according to initial bit budget. Notice that $n_{0}$ can either be computed from the optimal $\lambda$ chosen at startup using a bisection method on a typical R-D curve or estimated using the recursive formula (14). A bad estimation of the initial value will however not impair the quantization results, but rather displace them on the working rate-distortion curve. Finally, several coefficients could be used in the computation of $\tilde{\nu}$ to improve the accuracy of the estimation and avoid potential oscillatory effects.

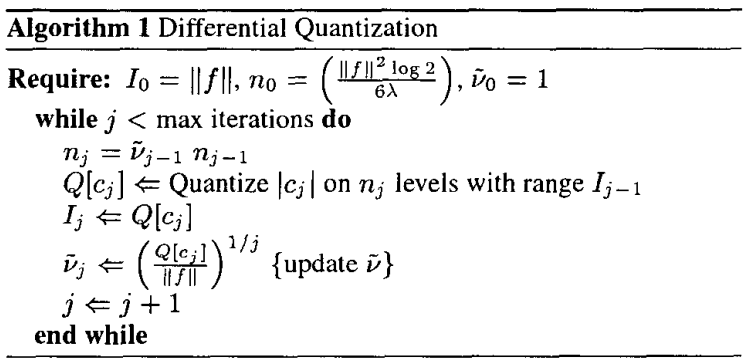

\section{EXPERIMENTAL RESULTS}

In this section we now compare the adaptive entropy-constrained quantization scheme with an exponential quantization scheme [11] used in Matching Pursuit coding [12]. The exponential quantization is clearly expected to provide better results than uniform quantization [7] due to the distribution of the MP coefficients. Both schemes, comparable in terms of complexity, are used for a posteriori MP coefficients quantization. In both cases, the distortion is reported to the coding rate estimated as the sum of the quantized coefficients entropy and the index average size. Note that the exponential quantization is similar to the one proposed in [12], where the deadzone is adapted to the statistics of the coefficients.

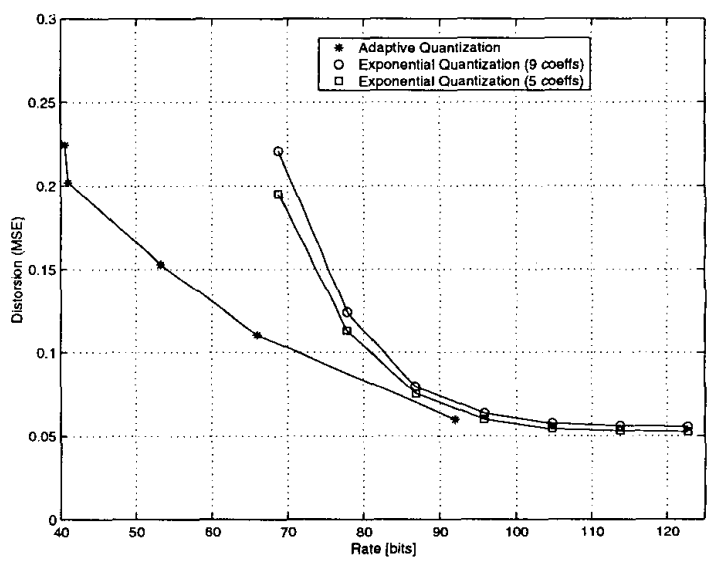

Fig. 1. Rate-distortion curve for adaptive and exponential quantization of random signals MP decompositions. 
Figure 1 shows the evolution of the MSE distortion versus the coding rate for both the adaptive entropy-constrained and the exponential quantization. 'The curve has been averaged on hundred decompositions of random signals of length 10 . The adaptive scheme clearly provides better results since it optimally distribute bits among MP coefficients. It moreover allows to reach much lower coding rates, since the number of coefficients is adaptively chosen according to the bit budget. Similar results are given in Figure 2 for the Matching Pursuit decomposition of the Lena image with a Gabor dictionary. The gain in MSE distortion for the adaptive quantization is mainly due to a fine quantization of the first and most energetic coefficients. In the case of the exponential quantization, the absolute arror on the first coefficients becomes very large, thus increasing the distortion according to Eq. (8).

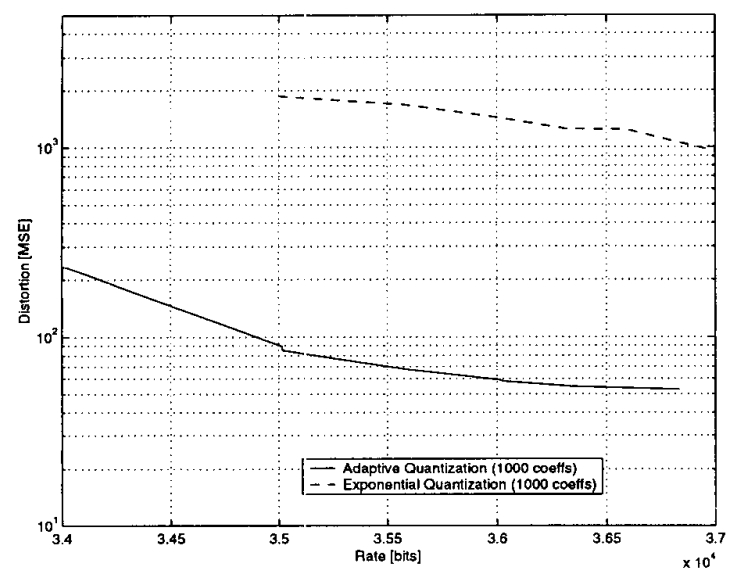

Fig. 2. Rate-distortion curve for adaptive and exponential quantization of the MP decomposition of the Lena image.

Finally, Figure 3 shows the reconstruction of the Lena image after adaptive and respectively exponential quantization for the same bit budget $(36.8 \mathrm{~kb})$. Clearly, the quality offered by adaptive quantization is much better than the one obtained with exponential quantization thanks to a finer quantization of the first MP coefficients.

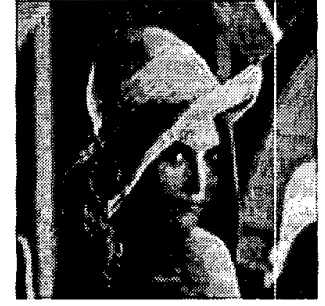

(a) $\operatorname{PSNR}=30.88 \mathrm{~dB}$

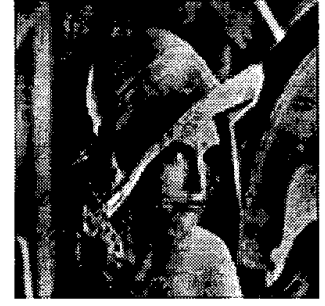

(b) PSNR $=18.33 \mathrm{~dB}$
Fig. 3. Reconstructed version of Lena reconstructed with $1000 \mathrm{Ga}$ bor atoms after adaptive and exponential coefficients quantization.

\section{CONCLUSION}

We proposed in this paper an adaptive entropy-constrained quantization scheme targeted for Matching Pursuit coefficients. This scheme is derived from an optimal entropy-constrained quantization by adapting to quantization parameters to the actual coefficients values. It truly outperforms an exponential quantization scheme thanks to the optimal distribution of the available bit budget across MP coefficients. Additionally, the adaptive scheme allows for a real-time quantization, while the exponential quantization scheme needs two passes to estimate the coefficients statistics before quantization.

\section{REFERENCES}

[1] Mallat S.G. and Zhang Z., "Matching Pursuits With TimeFrequency Dictionaries," IEEE Transactions on Signal Processing, vol. 41, no. 12, pp. 3397-3415, December 1993.

[2] Goyal V.K., Vetterli M. and Thao N.T., "Quantized Overcomplete Expansions in $R^{N}$ : Analysis, Synthesis and Algorithms," IEEE Transactions on Information Theory, vol. 44, no. 1, pp. 16-31, January 1998.

[3] Cvetkovic Z., "Source Coding with Quantized Redundant Expansions: Accuracy and Reconstruction," in Proceedings of the IEEE Data Compression Conference, 1999, pp. 344358.

[4] Goyal V.K. and Vetterli M., "Dependent coding in quantized matching pursuit," in Proceedings of the SPIE - Visual Communication and lmage Processing, 1997, vol. 3024, pp. $2-12$.

[5] Neff R. and Zakhor A., "Adaptive Modulus Quantizer Design for Matching Pursuit Video Coding," in Proceedings of the IEEE International Conference on Image Processing, 1999, vol. 2, pp. 81-85.

[6] Gharavi-Aikhansari M., "A model for entropy coding in matching pursuit," in Proceedings of the IEEE International Conference on Image Processing, 1998, vol. 1, pp. 778-782.

[7] Frossard P. and Vandergheynst P., "A Posteriori Quantized Matching Pursuit," in IEEE Data Compression Conference, Snowbird, UT, March 27-29 2001.

[8] Davis G., Mallat S. and Avellaneda M., "Adaptive Greedy Approximations," Journal of Constructive Approximations, vol. 13, pp. 57-98, 1997.

[9] Mallat S., A Wavelet Tour of Signal Processing, Academic Press, 2 edition, 1999.

[10] Frossard P. and Vandergheynst P., "Redundancy in NonOrthogonal Transforms," in IEEE International Symposium on Information Theory, Washington D.C., June 2001, submitted paper.

[11] Sullivan G.J., "Efficient Scalar Quantization of Exponential and Laplacian Random Variables," IEEE Transactions on Information Theory, vol. 42, no. 5, pp. 1365-1374, September 1996.

[12] Neff R. and Zakhor A., "Modulus Quantization for Matching-Pursuit Video Coding," IEEE Transactions on Circuits and Systems for Video Technology, vol. 10, no. 6, pp. 895-912, September 2000. 